\title{
Spotlight on isavuconazole in the treatment of invasive aspergillosis and mucormycosis: design, development, and place in therapy
}

This article was published in the following Dove Press journal:

Drug Design, Development and Therapy

\author{
Jeffrey D Jenks',* \\ Helmut JF Salzer ${ }^{2,3, *}$ \\ Juergen Prattes ${ }^{4,5, *}$ \\ Robert Krause $4,5, *$ \\ Dieter Buchheidt ${ }^{6, *}$ \\ Martin Hoenig| $1,3,7,8, *$ \\ 'Department of Medicine, University \\ of California San Diego, San Diego, \\ CA, USA; ${ }^{2}$ Division of Clinical \\ Infectious Diseases, Research Center \\ Borstel, Borstel, Germany; ${ }^{3}$ German \\ Center for Infection Research, Clinical \\ Tuberculosis Center, Leibniz Lung \\ Center, Borstel, Germany; ${ }^{4}$ Section \\ of Infectious Diseases and Tropical \\ Medicine, Medical University of Graz, \\ Graz, Austria; ${ }^{5} \mathrm{CBmed}$ - Center for \\ Biomarker Research in Medicine, \\ Graz, Austria; ${ }^{6}$ Department of \\ Hematology and Oncology, Mannheim \\ University Hospital, Heidelberg \\ University, Mannheim, Germany; \\ ${ }^{7}$ Division of Pulmonology, Medical \\ University of Graz, Graz, Austria; \\ ${ }^{8}$ Division of Infectious Diseases, \\ Department of Medicine, University \\ of California San Diego, San Diego, \\ CA, USA
}

*All authors contributed equally to this work
Correspondence: Martin Hoenigl Division of Infectious Diseases, Department of Medicine, University of California San Diego, 200 Dickinson Street, San Diego, CA 92103, USA $\mathrm{Tel}+$ I 6195435605

Email mhoenigl@ucsd.edu

\begin{abstract}
In recent decades, important advances have been made in the diagnosis and treatment of invasive aspergillosis (IA) and mucormycosis. One of these advances has been the introduction of isavuconazole, a second-generation broad spectrum triazole with a favorable pharmacokinetic and safety profile and few drug-drug interactions. Phase III trials in patients with IA and mucormycosis demonstrated that isavuconazole has similar efficacy to voriconazole for the treatment of IA (SECURE trial) and liposomal amphotericin B for the treatment of mucormycosis (VITAL trial with subsequent case-control analysis) and a favorable safety profile with significantly fewer ocular, hepatobiliary, and skin and soft tissue adverse events compared to voriconazole. As a result, recent IA guidelines recommend isavuconazole (together with voriconazole) as gold standard treatment for IA in patients with underlying hematological malignancies. In contrast to liposomal amphotericin B, isavuconazole can be safely administered in patients with reduced renal function and is frequently used for the treatment of mucormycosis in patients with reduced renal function. Updated guidelines on mucormycosis are needed to reflect the current evidence and give guidance on the use of isavuconazole for mucormycosis. Studies are needed to evaluate the role of isavuconazole for 1) anti-mold prophylaxis in highrisk patients, 2) salvage treatment for IA and mucormycosis, and 3) treatment for other mold infections such as Scedosporium apiospermum.
\end{abstract}

Keywords: TDM, plasma level, triazole, SECURE, VITAL, susceptibility, real life

\section{Introduction}

In recent decades, important advances have been made in the diagnosis of invasive aspergillosis (IA) and mucormycosis, ${ }^{1-8}$ although IA and mucormycosis still remain difficult to diagnose. ${ }^{9}$ In order to improve survival, empiric antifungal treatment is frequently initiated at early stages of infection where IA and/or mucormycosis are suspected, but mycological evidence is still missing. ${ }^{10}$

The introduction of broad spectrum antifungal agents such as voriconazole, posaconazole, and lipid-based formulations of amphotericin B has substantially improved the survival rates in patients with IA and mucormycosis over the past 15 years. ${ }^{11-14}$ Despite the overall improved safety profiles that came with these new treatment options, treatment of IA and mucormycosis remains complex due to unpredictable pharmacokinetic profiles, requiring therapeutic drug monitoring (TDM) for optimization of efficacy and prevention of adverse effects and multiple drug-drug interactions through the cytochrome P450 system. ${ }^{10,15-19}$ The recent introduction of isavuconazole, a new potent second-generation triazole with a favorable pharmacokinetic and safety profile, let to a reduction in drugdrug interactions. Similar efficacy of isavuconazole compared to other triazoles, may 
simplify treatment, and improve clinical practice. This review discusses the pharmacology of isavuconazole as well as its efficacy for the treatment of IA and mucormycosis in animal models, clinical studies, and real-life settings. We further discuss the prominent role of isavuconazole in recent guidelines and address future research questions.

\section{Pharmacology Chemistry and pharmacokinetics}

The prodrug of isavuconazole, isavuconazonium sulfate, is a water-soluble triazole precursor that can be administered both orally and intravenously. After administration, isavuconazonium sulfate is broken down by plasma esterases to the active component, isavuconazole, and an inactive cleavage product. This conversion occurs rapidly, with a half-life of less than a minute in vitro ${ }^{20,21}$ and with no detectable levels of prodrug or cleavage products following intravenous (IV) infusion after 30 minutes in healthy adults. ${ }^{22}$

Isavuconazole disrupts ergosterol biosynthesis by inhibiting cytochrome P450-dependent 14 $\alpha$-lanosterol demethylation, an essential component of ergosterol production. This mechanism is similar to that of the other azoles and significantly alters the structure and function of the fungal membrane, leading to cell death. ${ }^{20,21}$ In addition, isavuconazole consists of an [ $N$-(3-acetoxypropyl)- $N$-methylamino]carboxymethyl group side arm that orients the molecule to engage the triazole ring to the binding pocket of the fungal CYP51 protein, conferring activity against some pathogens resistant to other azoles, including itraconazole, voriconazole, and posaconazole. ${ }^{23}$

Unlike the other second-generation triazoles, isavuconazole is highly water soluble and does not require the addition of a beta-cyclodextrin to its IV formulation to facilitate solubility, ${ }^{24}$ eliminating the potential for nephrotoxicity from the cyclodextrin vehicle. Isavuconazole has a large volume of distribution and is highly protein bound with $~ 98 \%$ bound to proteins, mostly to albumin. ${ }^{23}$ The absorption and serum concentrations of isavuconazole are not dependent on the timing of food intake, so it can be taken with or without food. ${ }^{25}$ The serum concentrations of isavuconazole are not reduced in patients with mucositis, ${ }^{26}$ and the bioavailability is not affected with concomitant administration of medications that suppress gastric acid production, so it can be taken with proton pump inhibitors or other medications that affect gastric acid secretion. ${ }^{27}$

Key pharmacokinetic characteristics of isavuconazole are displayed in Table 1. Conventional dosing is expressed in terms of milligram equivalent of the active compound,
Table I Key pharmacokinetic characteristics of isavuconazole

\begin{tabular}{ll}
\hline Characteristics & Isavuconazole \\
\hline Dosage & $200 \mathrm{mg}$ q8h day I+2; \\
& $200 \mathrm{mg}$ q24h maintenance \\
Resorption po/IV & po $=$ IV \\
Oral intake with/without food & No influence \\
Half-life, hours & II0-II5 \\
Oral bioavailability & $98 \%$ \\
Linear pharmacokinetics & Yes \\
Central nervous system penetration & High (animal model) \\
Renal excretion & $<1 \%$ \\
Metabolism & CYP3A4/5 \\
Exposure-response relationship & No \\
Intra-patient variability & Low \\
Inter-patient variability & Medium to low \\
\hline
\end{tabular}

Abbreviations: IV, intravenous; po, per oral; q8h, every 8 hours; q24h, every 24 hours.

isavuconazole. The recommended dose for both oral and IV administration is a loading dose of $200 \mathrm{mg}$ of isavuconazole (equivalent to $372 \mathrm{mg}$ of isavuconazonium sulfate) every 8 hours for six doses given over a 48-hour period, followed by a maintenance dose of $200 \mathrm{mg}$ of isavuconazole daily.

In healthy adult volunteers, isavuconazole demonstrates dose-proportional pharmacokinetics with low inter-subject and intra-subject variabilities. However, high body mass index has been shown to be associated with higher clearance of isavuconazole. ${ }^{28}$ The bioavailability of isavuconazole is excellent with $\sim 98 \%$ of the active drug reaching circulation. In healthy volunteers, plasma concentrations reached a maximum concentration after 2-3 hours of oral administration and after 1 hour of IV administration. ${ }^{20,21}$ In the Phase III, double-blind, multicenter SECURE trial, 527 patients with IA or other filamentous fungi were randomized to receive either voriconazole or isavuconazole. There was low intra-subject variability and narrow trough-level distribution in the isavuconazole group, suggesting that routine TDM is not necessary. ${ }^{29,82}$ Terminal half-life elimination of isavuconazole ranges from 100 to 115 hours, ${ }^{20,21}$ and there is no clear exposure-response relationship, ${ }^{82} \mathrm{ie}$, low plasma levels did not clinically translate into a worse response. As a consequence, isavuconazole TDM is not routinely recommended in the European Conference on Infectious in Leukemia (ECIL-6) guidelines ${ }^{30}$ and is only a CIII recommendation in the European Society of Clinical Microbiology and Infectious Diseases (ESCMID)/European Confederation of Medical Mycology (ECMM) 2018 guidelines. ${ }^{31}$ In contrast, TDM is an AI-AII recommendation for voriconazole (to ensure efficacy and avoid toxicity ${ }^{16}$ ) and posaconazole (to ensure efficacy ${ }^{18,32}$ ) when used for the treatment of invasive mold infections. 
Isavuconazole is thought to be widely distributed in nearly all tissues, including the liver, lungs, eyes, kidneys, bone, nasal mucosa, and brain. In rat animal models, mean plasma concentrations of isavuconazole were similar to mean brain concentrations after a single oral dose of isavuconazole $(25 \mathrm{mg} / \mathrm{kg}) .{ }^{33} \mathrm{In} 2$ patients with fungal meningitis associated with contaminated epidural steroid injections who failed treatment with both liposomal amphotericin and voriconazole, salvage isavuconazole treatment led to complete resolution of infection with negative follow-up cerebral spinal fluid (CSF) analysis at 1, 3, and 6 months posttreatment. Both patients remained infection-free at 12 months following completion of therapy. This occurred despite low CSF levels of isavuconazole compared to plasma levels, ${ }^{34}$ suggesting that the CSF levels may not adequately reflect the high brain tissue concentrations seen in animal studies.

\section{Metabolism and elimination}

There are negligible urinary concentrations of isavuconazole following oral administration with only slightly higher urinary concentrations following IV dosing. ${ }^{20,21}$ Thus, isavuconazole dosage does not need to be reduced in patients with reduced renal function. ${ }^{28}$ Neither the area under the curve (AUC) nor the maximum serum concentration $\left(C_{\max }\right)$ is affected in renal impairment, so no dose adjustment is recommended in individuals with mild, moderate, or severe renal impairment including end-stage renal disease (ESRD). Isavuconazole is not thought to be dialyzable. ${ }^{24}$ Metabolism of isavuconazole takes place in the liver by cytochrome $\mathrm{P} 450$ (CYP) isoenzymes, particularly CYP3A4 and CYP3A5. Although patients with liver disease have higher exposure to isavuconazole, no dose adjustment is recommended in individuals with mild to moderate hepatic impairment (ChildPugh classes A and B). There are no data on individuals with severe liver disease (Child-Pugh class C).

Isavuconazole is a pregnancy class $\mathrm{C}$ drug and should not be given to pregnant women unless the potential benefit to the patient outweighs the risk to the fetus. The drug is excreted in the milk of lactating rats, and so it should be avoided in women who are breastfeeding. ${ }^{24}$

\section{Pharmacodynamics}

\section{In vitro susceptibility profile of isavuconazole}

Isavuconazole appears to be highly active against all Candida species including species such as C. glabrata and C. kru$s e i$, which are inherently less susceptible to other azoles such as fluconazole. ${ }^{35,36}$ In one study of 296 isolates from bloodstream infections obtained over a period of 10 years, isavuconazole demonstrated greater potency than fluconazole against every organism tested and was as active as itraconazole and voriconazole. ${ }^{37}$ The minimum inhibitory concentration (MIC) values for $50 \%$ inhibitory $\left(\mathrm{MIC}_{50}\right)$ and $90 \%$ inhibitory $\left(\mathrm{MIC}_{90}\right)$ of all isolates were $<0.5$ and $<2.0 \mathrm{mg} / \mathrm{L}$, respectively, for all major Candida species (Table 2). In another study of 1,358 isolates of Candida encompassing 11 different species, isavuconazole showed greater potency than posaconazole. ${ }^{38}$ Isavuconazole also has an excellent activity against Cryptococcus gattii and $C$. neoformans, with comparable efficacy to posaconazole and voriconazole and greater efficacy against some isolates with reduced fluconazole susceptibilities. This was demonstrated in a study of 128 cryptococcal isolates. The $\mathrm{MIC}_{50}$ and $\mathrm{MIC}_{90}$ of 86 isolates of C. neoformans were $<0.015$ and $0.6 \mathrm{mg} / \mathrm{L}$, respectively, and those of 42 isolates of $C$. gattii were 0.03 and $0.06 \mathrm{mg} / \mathrm{L}$, respectively. ${ }^{39}$ Isavuconazole also has the activity against other yeast, including Trichosporon spp..$^{40}$ In addition, isavuconazole showed also the activity against Coccidioides (MICs: $0.06-0.12 \mu \mathrm{g} / \mathrm{mL} ; \mathrm{n}=6$ ), Histoplasma (MICs: $0.03 \mu \mathrm{g} / \mathrm{mL} ; \mathrm{n}=2$ ), and Paracoccidioides (MIC: $0.001 \mu \mathrm{g} / \mathrm{mL} ; \mathrm{n}=1) .{ }^{87}$

Most importantly, isavuconazole has an outstanding activity against the most common species of Aspergillus. In a study of 118 isolates of Aspergillus spp., including $A$. fumigatus, A. terreus, A. flavus, and A. niger, isavuconazole demonstrated the activity against all four Aspergillus species including strains resistant to itraconazole, caspofungin, or amphotericin B..$^{41,42}$ In addition, isavuconazole also demonstrated the activity against $A$. lentulus ${ }^{43}$ Importantly, isavuconazole has a very good activity against Mucorales, ${ }^{44}$ although there is an unmet need of harmonization between methods that determine mucorales's MICs and also Scedosporium apiospermum but limited in vitro activity against Fusarium species and Lomentospora prolificans (Table 3). ${ }^{45}$

Table 2 In vitro susceptibilities of isavuconazole against common medically important yeast

\begin{tabular}{llll}
\hline Organism & MIC $_{50}(\mathbf{m g} / \mathbf{L})$ & MIC $_{90}(\mathbf{m g} / \mathbf{L})$ & References \\
\hline Candida albicans & 0.015 & $<0.015-0.03$ & $35-38,40,86$ \\
Candida glabrata & $<0.015-0.5$ & $0.2-2.0$ & $35-38,40,86$ \\
Candida krusei & $<0.015-0.5$ & $<0.015-1.0$ & $35-38,86$ \\
Candida parapsilosis & $<0.015-0.06$ & $0.023-0.12$ & $35-38,40,86$ \\
Candida tropicalis & $<0.015-0.06$ & $<0.015-0.5$ & $35-38,40,86$ \\
Cryptococcus neoformans & $<0.015-0.6$ & $<0.015-0.12$ & $35,36,39$ \\
Cryptococcus gattii & $0.03-0.06$ & $0.06-0.125$ & $38,39,43$ \\
Trichosporon spp. & 0.06 & 0.5 & 40 \\
\hline
\end{tabular}

Abbreviations: $\mathrm{MIC}_{50}$, minimum inhibitory concentration for $50 \%$ inhibitory; $\mathrm{MIC}_{90}$, minimum inhibitory concentration for $90 \%$ inhibitory. 
Table 3 In vitro susceptibilities of isavuconazole against medically important molds

\begin{tabular}{llll}
\hline Organism & MIC $_{50}(\mathbf{m g} / \mathbf{L})$ & $\mathbf{M I C}_{90}(\mathbf{m g} / \mathbf{L})$ & References \\
\hline Aspergillus fumigatus & $0.25-1.0$ & $0.5-2.0$ & $36,42,45$ \\
Aspergillus terreus & $0.38-0.5$ & 0.5 & 42,45 \\
Aspergillus flavus & $0.38-2.0$ & $0.5-2.0$ & $36,42,44,45$ \\
Aspergillus niger & 0.5 & $0.75-2.0$ & 42,45 \\
Fusarium spp. & $8.0->32$ & $>8.0->32$ & 44 \\
Scedosporium & $>32$ & $>32$ & 45 \\
apiospermum & & & \\
Mucor spp. & 2 & $>32$ & 44,45 \\
Rhizopus spp. & 1 & $>32$ & 44,45
\end{tabular}

Abbreviations: $\mathrm{MIC}_{50}$, minimum inhibitory concentration for $50 \%$ inhibitory; $\mathrm{MIC}_{90}$, minimum inhibitory concentration for $90 \%$ inhibitory.

\section{Safety and tolerability}

Isavuconazole is thought to be safe and is generally well tolerated. In early single-ascending dose and multiple-dose studies, the most common reported side effects were upper respiratory symptoms, headache, and mild gastrointestinal symptoms including abdominal pain, nausea, and diarrhea. ${ }^{20,21}$ Similar adverse events were found in a Phase II multicenter clinical trial of 160 participants with uncomplicated esophageal candidiasis, in which subjects were randomized to three oral doses of isavuconazole or oral fluconazole. A minority of patients, $18.2 \%$, experienced at least one medication-related adverse event, with anemia and gastrointestinal side effects including diarrhea and nausea being the most common. ${ }^{46}$ The SECURE trial found that although drug-related adverse events were common, there were significantly fewer adverse events in the isavuconazole group than in the voriconazole group ( $42 \%$ versus $60 \%$, respectively) ${ }^{29}$ This was true in particular for eye disorders (number needed to treat [NNT] with isavuconazole $=9$ to avoid the adverse event), skin and subcutaneous tissue disorders ( $\mathrm{NNT}=11)$, and hepatobiliary disorders $(\mathrm{NNT}=14){ }^{29}$

In the early dosing trials, no serious adverse events were reported that were thought to be related to isavuconazole, although one study participant developed a mild elevation of liver transaminases leading to discontinuation of the study drug with subsequent normalization of liver function tests. ${ }^{20,21}$ In a Phase II trial, 2 patients in the isavuconazole arm experienced serious adverse events (atrioventricular block and tuberculosis pleurisy). ${ }^{46}$ There was a lower frequency of drug-related hepatobiliary adverse events with isavuconazole compared to voriconazole ( $2 \%$ versus $10 \%$, respectively) in the SECURE trial and fewer eye disorders in those that took isavuconazole compared to voriconazole (visual impairment, 4/257 [2\%] versus 19/259 [7\%], photophobia, $2 / 257$ [1\%] versus $6 / 259$ [2\%], reduced visual acuity, $1 / 257[<1 \%$ ] versus $6 / 259$ [2\%], and retinal hemorrhage, $0 / 257[0 \%]$ versus $5 / 259$ [2\%]). Furthermore, permanent drug discontinuation was lower for isavuconazole than for voriconazole ( $8 \%$ versus $14 \%$, respectively). ${ }^{29} \mathrm{QT}$ interval shortening can occur with isavuconazole, and this is thought to be dose-dependent, although there is no evidence of associated cardiac risk. Still, its use is contraindicated in patients with familial short QT syndrome (SQTS). ${ }^{47,48}$

Thus, isavuconazole seems to be at least as well tolerated as voriconazole and does not appear to be associated with some of the common side effects of voriconazole such as vision changes, photosensitivity, and hepatotoxicity. Although isavuconazole, like other azoles, has been associated with hepatotoxicity, this is generally reversible and generally does not require drug discontinuation. ${ }^{24}$ Infusion reactions including hypotension, dyspnea, chills, dizziness, and paresthesias have been reported during infusion and may require discontinuation of the infusion. ${ }^{24}$ Following dilution, IV isavuconazole may form precipitate from the insoluble isavuconazole, so it is recommended that isavuconazole should be administered through an in-line filter. ${ }^{24}$

\section{Drug-drug interactions}

Isavuconazole is a sensitive substrate of CYP3A4, so medications that inhibit this enzyme can increase isavuconazole levels and should be used cautiously if used concurrently. Isavuconazole use with strong CYP3A4 inhibitors such as ketoconazole and high-dose ritonavir as well as strong CYP3A4 inducers such as long-acting barbiturates, St John's wort (Hypericum perforatum), carbamazepine, and rifampin is contraindicated. Isavuconazole is a moderate inhibitor of CYP3A4 and can increase drug levels of atorvastatin, cyclosporine, sirolimus, tacrolimus, midazolam, and mycophenolate mofetil; concurrent use of these drugs should be closely monitored. Although studies have shown that isavuconazole may be favorable because of limited drug-drug interactions compared to other second-generation azoles when used together with these immunosuppressants, drug levels of cyclosporine, sirolimus, and tacrolimus, if used concurrently with isavuconazole, should be monitored and if necessary adapted. ${ }^{49}$ Isavuconazole is a mild inhibitor of P-glycoprotein (P-gp), so when used with digoxin, serum digoxin levels should be routinely monitored. ${ }^{24}$ Given the potential for isavuconazole to shorten the QTc interval, it should be prescribed with caution when prescribed with medications known to shorten the QTc interval. ${ }^{47,48}$

\section{Efficacy against aspergillosis}

IA is the most frequently encountered invasive mold infection in immunocompromised patients, with mortality rates of $30 \%-40 \% .6,7,50$ 


\section{In vivo antifungal susceptibility profiles based on animal models}

Preclinical studies exploring the efficacy of isavuconazole for IA treatment investigated several animal models with A. fumigatus, A. flavus, and A. terreus infections, with a particular focus of $A$. fumigatus in invasive pulmonary aspergillosis models. Petraitis et al ${ }^{51}$ investigated several dosing regimens of isavuconazole in severely neutropenic rabbits, ${ }^{52}$ who were infected with the NIH4215 A. fumigatus strain via endotracheal inoculation. Following infection, these rabbits received an oral loading dose of isavuconazole $(90 \mathrm{mg} / \mathrm{kg})$ followed by oral isavuconazole $(20,40$, or $60 \mathrm{mg} / \mathrm{kg}$ once daily). Rabbits treated with voriconazole $(15 \mathrm{mg} / \mathrm{kg}$ orally twice daily) and untreated rabbits were used as control groups. Results showed an improved survival in all the three isavuconazole groups compared to untreated rabbits, as well as an improved survival in rabbits treated with isavuconazole (40 mg/ $/ \mathrm{kg}$ [six of nine surviving] and $60 \mathrm{mg} / \mathrm{kg}$ [four of nine surviving]) compared to rabbits in the voriconazole group (zero of eight surviving). In addition, compared to the control and voriconazole groups, rabbits in the isavuconazole groups showed reduced fungal burdens in lung tissue as well as lower serum and bronchoalveolar lavage fluid galactomannan levels and serum 1,3- $\beta$-D-glucan levels. A similar model compared the activity of isavuconazole in mice infected with a different A. fumigatus strain (AF293) ${ }^{52}$ In this study, mice were treated with either subcutaneous isavuconazole (15 and $30 \mathrm{mg} / \mathrm{kg}$ twice daily) or oral posaconazole with a control group of untreated mice. While posaconazole improved survival and decreased fungal lung burden, isavuconazole neither improved survival nor reduced fungal burden in lung tissue, compared to untreated mice. Notably, in this study, isavuconazole concentrations in lung tissue were low $(<1 \mu \mathrm{g} / \mathrm{mL})$, possibly due to subcutaneous administration or due to a higher MIC of the A. fumigatus strain. Furthermore, the combination of a lower AUC of isavuconazole and higher MIC may have resulted in a lower AUC/MIC ratio, which is a strong predictor for treatment efficacy in animal studies. ${ }^{53,54}$

Isavuconazole activity was also investigated against A. flavus in mice models. ${ }^{42}$ In this study, mice were treated with cyclophosphamide to induce a neutropenic state and were infected intravenously with A. flavus (strain AFL8). Different doses of isavuconazole were compared to voriconazole, itraconazole, and caspofungin. Dosing of antifungals was done 2 hours pre-infection, 4 hours post-infection, or 24 hours post-infection as follows: oral isavuconazole (3, 6 , or $15 \mathrm{mg} / \mathrm{kg}$ ) three times daily at day 1 and day 2 followed by twice daily dosing; oral voriconazole $(10 \mathrm{mg} / \mathrm{kg})$ once daily; oral itraconazole $(10 \mathrm{mg} / \mathrm{kg})$ three times daily on day 1 and day 2 followed by twice daily dosing; and IV caspofungin $(1 \mathrm{mg} / \mathrm{kg})$ once daily. End points were survival after 14 days of infection and fungal tissue burden (kidneys, liver, lungs, brain, and spleen) obtained by fungal culture and quantitative real-time polymerase chain reaction (PCR). In the pre-infection cohorts dosed with isavuconazole $(6 \mathrm{mg} /$ $\mathrm{kg}$ ) and caspofungin, there was a $100 \%$ survival rate at day 14 versus a $0 \%$ survival rate at day 14 in the voriconazole cohort. In the 4-hour post-infection cohorts dosed with isavuconazole (15 mg/kg), voriconazole, and caspofungin, there was an $83 \%$ survival rate (five out of six animals survived). In the 24-hour post-infection groups dosed with isavuconazole (15 and $30 \mathrm{mg} / \mathrm{kg}$ ) and caspofungin, there was a $100 \%$ survival rate. ${ }^{42}$

In a similar study investigating mice with disseminated aspergillosis infected with A. fumigatus, A. flavus, or $A$. terreus, various doses of isavuconazole were compared to amphotericin B, caspofungin, voriconazole, and an untreated control group. The primary end point was fungal clearance. Isavuconazole and comparator antifungals were found to be similarly efficacious in clearing fungal burden from the kidneys of mice infected with Aspergillus spp., including the A. flavus strain and the A. terreus strain (AT49), showing a high minimal fungicidal concentration. ${ }^{54}$

\section{Clinical efficacy of isavuconazole against IA}

After showing in vitro activity against Aspergillus, isavuconazole entered clinical studies (Phase II and Phase III). Safety, efficacy, and dosing regimens of isavuconazole were investigated in two Phase II studies. ${ }^{46,55}$ Based on these results, a Phase III, double-blind, randomized, non-inferiority trial was performed comparing the efficacy and safety of isavuconazole versus voriconazole as primary treatment of invasive fungal diseases caused by Aspergillus spp. or other filamentous molds (SECURE trial).$^{29}$ The primary end point of this study was all-cause mortality at day 42 of treatment in intention-to-treat (ITT) analysis. Secondary end points included overall treatment response at the end of treatment; all-cause mortality at day 84 of treatment; a composite of clinical, mycological, and radiological responses; and safety and tolerability profiles. A total of 527 patients were randomized to receive either isavuconazole $(n=263)$ at a dose of $200 \mathrm{mg}$ intravenously three times daily on day 1 and day 2 , followed by $200 \mathrm{mg}$ orally or intravenously once daily, or voriconazole $(\mathrm{n}=264)$ at doses of $6 \mathrm{mg} / \mathrm{kg}$ twice daily intravenously on day 1 and $4 \mathrm{mg} / \mathrm{kg}$ intravenously twice daily on day 2 , followed by either IV treatment with $4 \mathrm{mg} / \mathrm{kg}$ twice daily or $200 \mathrm{mg}$ orally twice daily. A total of 516 patients 
(256 per group) received at least one dose of isavuconazole or voriconazole. Out of the 516 patients, a data-reviewing committee identified 272 patients as having proven or probable invasive mold disease (modified ITT [mITT] population) and 231 as having proven or probable IA (mycological ITT [myITT] population). Aspergillus spp. were identified as the primary pathogen in $\sim 30 \%$ of the mITT population, with $A$. fumigatus being the most common pathogen. Mixed mold infections were observed in $2 \%$ of the mITT population.

Regarding the primary end point of all-cause mortality at day 42, isavuconazole was found to be non-inferior to voriconazole in the ITT population (19\% mortality in the isavuconazole group versus $20 \%$ in the voriconazole group; adjusted treatment difference $-1.0 \%, 95 \%$ confidence interval: $-7.8 \%$ to $5.7 \%$ ). Results were similar for the mITT and myITT populations (20\% versus $23 \%$ and $19 \%$ versus $22 \%$, respectively). Overall treatment response at the end of treatment - including clinical, mycological, and radiological responses - and mortality at day 84 were similar in both groups. However, patients receiving isavuconazole developed significantly fewer hepatobiliary, eye, and skin disorders. In addition, treatment discontinuation due to drugrelated events was significantly lower in the isavuconazole group. Thus, the SECURE study showed isavuconazole to be non-inferior to voriconazole for the treatment of invasive mold diseases due to Aspergillus spp. or other filamentous fungi, with a lower rate of adverse events. Based on this study, the European Medicines Agency (EMA) and the US Food and Drug Administration (FDA) licensed isavuconazole for the treatment of IA.

\section{Guideline recommendations}

Both isavuconazole and voriconazole are recommended as first-line treatment for IA in patients with hematological malignancies in the ESCMID/ECMM 2018 guidelines (AI recommendation) $)^{31}$ and the ECIL-6 guidelines (AI-AII recommendation $)^{30}$ (Table 4 ). This contrasts to liposomal

Table 4 Guideline recommendations for the treatment of invasive (pulmonary) aspergillosis in hematological malignancy patients

\begin{tabular}{llll}
\hline Guideline & Isavuconazole & Voriconazole & $\begin{array}{l}\text { Liposomal } \\
\text { amphotericin B }\end{array}$ \\
\hline ECIL-6 & Al & $\mathrm{Al}$ & $\mathrm{BI}$ \\
ESCMID/ & Al-All & Al-All & Bll \\
ECMM 201831 & & Al & All \\
IDSA 2016 & All & A
\end{tabular}

Abbreviations: ECIL-6, European Conference on Infectious in Leukemia; ECMM, European Confederation of Medical Mycology; ESCMID, European Society of Clinical Microbiology and Infectious Diseases; IDSA, Infectious Diseases Society of America. amphotericin B, which has BI and BII recommendations in both European guidelines. The 2016 Infectious Diseases Society of America (IDSA) guidelines differ slightly from the European guidelines, giving voriconazole an AI recommendation and both isavuconazole and liposomal amphotericin B an AII recommendation. ${ }^{56}$ Given the favorable safety profile of isavuconazole compared to voriconazole, isavuconazole may become the treatment of choice for IA in patients with hematological malignancies.

Given a lack of data for the treatment of IA with isavuconazole in patients without hematological malignancies, voriconazole remains the sole first-line treatment option in this context (voriconazole has an AIII recommendation in the ESCMID 2018 guidelines). ${ }^{31}$ There are also limited data on isavuconazole as salvage treatment in patients with and without hematological malignancies, so posaconazole, liposomal amphotericin $\mathrm{B}$, and voriconazole remain the antifungal agents of choice in these settings. ${ }^{30,31}$ Limited data exist on the efficacy of isavuconazole for the treatment of chronic pulmonary aspergillosis (CPA). However, given the low MICs of Aspergillus spp. and the favorable safety profile of isavuconazole, it has been recommended as a third-line treatment for CPA in those that failed treatment with voriconazole and itraconazole. ${ }^{54}$

\section{Isavuconazole: efficacy against mucormycosis}

Mucormycosis is a rare but life-threatening disease that is caused by various species of the order Mucorales. The infection not only occurs primarily in patients with hematological malignancies, including patients following hematopoietic stem cell transplantation, but also occurs in other immunocompromised patients including those with iron overload and diabetes mellitus. ${ }^{57}$

If not promptly diagnosed and treated, mucormycosis is associated with $>90 \%$ mortality. ${ }^{57}$ Preventing such outcomes requires appropriate clinical awareness and a high index of suspicion since current diagnostic modalities have limited reliability and are often invasive. Positive clinical outcomes require early antifungal administration and may require extensive surgery.

Until the approval of isavuconazole, liposomal formulation of amphotericin B (lipAmB) has been the first-line antifungal treatment of mucormycosis, whereas posaconazole has been recommended for lipAmB intolerant or refractory cases. ${ }^{58,59}$ The addition of surgical debridement and, if possible, the correction of the underlying immunosuppression and control of diabetes have led to a $40 \%$ reduction in 
mortality over the past decade. ${ }^{58}$ However, the use of antifungals is often restricted given the common adverse effects of lipAmB, which often cannot be used in patients with renal impairment, and a lack of data supporting first-line treatment with posaconazole.

Given the low prevalence and wide clinical and microbiological spectrum of infections caused by mucormycetes, recommendations for the treatment of these infections had been mostly based on registry data, small prospective noncontrolled studies, and small retrospective trials. ${ }^{59}$ In the Deferasirox-AmBisome Therapy for Mucormycosis study, 20 patients suffering from mucormycosis were randomized to receive $\operatorname{lip} \mathrm{AmB}$ combined with deferasirox, an iron chelating agent, or lipAmB and placebo. Patients treated with adjunctive deferasirox had increased mortality, and the trial was discontinued. ${ }^{60}$ In the AmBizygo study, a single-arm Phase II trial, 34 patients were treated with $10 \mathrm{mg} / \mathrm{kg}$ per day of lipAmB for disseminated mucormycosis. The mortality rate at 12 weeks was $38 \%$, but $40 \%$ of patients developed significant nephrotoxicity. ${ }^{61}$

Isavuconazole has shown convincing in vitro and preclinical in vivo data - including linear pharmacokinetics and high bioavailability - and good activity against a wide spectrum of fungal pathogens including mucormycetes. ${ }^{62-64}$ In addition, isavuconazole was well tolerated by healthy volunteers as well as patients undergoing chemotherapy for acute leukemia in Phase II studies. The VITAL Phase III study was a single-arm open-label study of isavuconazole for the treatment of disseminated mucormycosis, combined with a case-control analysis comparing matched cases treated with $\operatorname{lipAmB}$ from the Fungiscope registry ${ }^{65}$ and assessed the safety and efficacy of isavuconazole for the treatment of infections caused by mucormycetes and other rare molds. The primary objective was to assess the efficacy of isavuconazole, and the case-control analysis evaluated the mortality outcomes in patients who took isavuconazole compared to lipAmB. ${ }^{66}$ Adult patients ( $\geq 18$ years) were recruited from 34 centers worldwide from April 2008 to June 2013. The study defined primary treatment of mucormycosis as $\leq 4$ days of previous systemic antifungal therapy. Patients were eligible if they had been refractory or intolerant to other antifungal drugs. Disseminated mucormycosis was defined as involving $>1$ noncontiguous body region. The diagnostic certainty of invasive fungal disease using European Organization for Research and Treatment of Cancer/Mycoses Study Group criteria was evaluated by an independent data review committee, and mucormycosis infection was defined as "proven" if there was histopathological evidence of disease or growth on culture from sterile body sites or defined as "probable" in the case of pathogen growth from respiratory specimens in patients with lung infiltrates and/or pneumonia and no alternative etiology for infection. Participants received an IV or oral loading dose of isavuconazole (200 mg), every 8 hours for six doses, followed by isavuconazole $(200 \mathrm{mg}$ daily). At day 42, day 84, and at the end of treatment, the investigators assessed and documented clinical, radiological, and mycological responses.

To assess the clinical efficacy of isavuconazole for the treatment of mucormycosis, the investigators performed a matched case-control analysis using data from the FungiScope registry, which is a global, anonymized database of rare invasive fungal diseases. In this matched case-control analysis, patients receiving primary treatment with isavuconazole were matched with FungiScope patients who had been treated with first-line therapy with amphotericin B. The primary study end point was defined as overall response at day 42. Secondary end points included all-cause mortality rates at day 42 and day 84, and clinical, radiological, and mycological responses at day 42 , day 84 , and at the end of treatment.

Of the 149 patients with invasive fungal infections (IFIs) enrolled, 37 had proven $(86 \%)$ or probable (14\%) mucormycosis. Twenty-one patients were treated with isavuconazole first-line, 11 for refractory disease, and 5 after intolerance to other antifungals. By treatment day 84, complete response was documented in $2(5 \%)$ of 37 patients, partial responses in 5 patients $(14 \%)$, and stable disease in 11 patients $(30 \%)$. All-cause mortality was 43\% (16 of 37 patients).

The reasons for discontinuation of isavuconazole treatment were death (11 patients [30\%]), adverse events (6 patients [16\%]), noncompliance (4 patients [11\%]), treatment response failure (2 patients [5\%]), and decision of an investigator (1 patient [3\%]). Adverse events that led to cessation of treatment were relapse or progression of malignant disease (2 patients), liver injury (2 patients), Gram-negative bacteremia (1 patient), and nausea ( 1 patient). The adverse events reported by $>10 \%$ of patients were similar to those reported in the SECURE trial, mostly gastrointestinal symptoms (nausea, vomiting, and diarrhea).

In the matched case-control analysis, 33 patients treated with amphotericin B in the FungiScope registry were matched as controls to 21 VITAL study patients who received isavuconazole for primary antifungal treatment. Survival probability through day 84 was similar between FungiScope control cases $(50 \%)$ and VITAL study patients $(57 \%)$. 
A correlation between fungal isolate MICs, isavuconazole plasma concentrations, and key outcomes could not be identified.

Apart from the VITAL study, isavuconazole has been reported as a successful salvage therapy for mucormycosis in single case reports. ${ }^{67-70}$

\section{Guideline recommendations}

In summary, the VITAL study showed that isavuconazole was efficacious for both primary and salvage therapies (refractory or intolerant to other antifungals) for the treatment of mucormycosis infections, with overall end-oftreatment complete and partial responses of $32 \%$ for primary treatment and $36 \%$ for the treatment of mucormycosis refractory to other antifungals. These findings were similar to response rates reported for $\operatorname{lip} A m B$, although isavuconazole lacks the dose-limiting nephrotoxicity of lipAmB. Overall, isavuconazole was well tolerated and safe, and toxicity was an uncommon cause for treatment discontinuation. Based on these results, the FDA has licensed isavuconazole for the treatment of mucormycosis infections, whereas the EMA has licensed isavuconazole for use in cases where AmB cannot be used, so AmB formulations likely continue to be the first-line treatment for mucormycosis in Europe. Still, isavuconazole and posaconazole may be viable options to AmB in patients who do not tolerate liposomal amphotericin $\mathrm{B}$ and those with reduced renal function. Updated guidelines are needed that define the role of isavuconazole for the treatment of mucormycosis.

\section{Real-life data}

Clinical decisions should be based on the guideline recommendations that reflect an expert consensus based on available evidence. However, in real-world scenarios, the choice of an appropriate antifungal agent may be complicated by several factors including serious drug allergies such as anaphylaxis, fungal resistance, nephrotoxicity, drug-drug interactions, and the need for a reliable oral formulation, as demonstrated in a recently published case report. ${ }^{10}$ Thus, isavuconazole may find greater use despite the aforementioned recommendations. Although voriconazole remains the treatment of choice for central nervous system (CNS) aspergillosis due to its superior penetration into the $\mathrm{CNS},{ }^{71,72}$ isavuconazole may be the best second-line alternative, despite the limited amount of data that are available on isavuconazole efficacy in cases of CNS infection.

Data on the use of isavuconazole as antifungal prophylaxis are very limited. In the recently published 2017 update of the recommendations of the Infectious Diseases Working Party (AGIHO) of the German Society for Haematology and Medical Oncology (DGHO), isavuconazole was graded as CII in patients with neutropenia ( $<500$ cells $/ \mu \mathrm{L}>7$ days $)$, a lower grade than other antifungal drugs due to limited data supporting its use as prophylaxis. ${ }^{73}$ Clinical studies investigating prophylaxis with isavuconazole in patients undergoing allogeneic hematopoietic stem cell transplant (ClinicalTrials. gov identifier: NCT03149055) and in patients with acute myeloid leukemia (AML) or myelodysplastic syndrome and neutropenia (ClinicalTrials.gov identifier: NCT03019939) are currently recruiting patients.

A small Phase II dose escalation study supports the safety and tolerability of IV isavuconazole prophylaxis in patients with AML who had undergone chemotherapy and had preexisting/expected neutropenia. ${ }^{55}$ After a loading dose, maintenance isavuconazole was administered at $200 \mathrm{mg}$ (low-dose cohort) and $400 \mathrm{mg}$ (high-dose cohort) once daily for 23 days. Eighteen of 20 patients who completed the study were classified as a treatment success, whereas 2 patients in the low-dose cohort developed possible IFI. Four patients discontinued the study due to adverse events (two in each cohort), but no drug-related treatment-emergent adverse event was considered as life threatening. Most adverse events were classified as mild to moderate in severity including headache and rash (55\% high-dose cohort versus 25\% lowdose cohort). Cardiac QTc prolongation under isavuconazole treatment was not observed during this study.

Unlike other azoles which can result in QTc interval prolongation, QTc interval shortening has been reported with isavuconazole. In a large Phase I, randomized, doubleblind study, healthy individuals who received isavuconazole $(n=69)$ developed a shortened QTc interval, which was both dose and plasma concentration dependent. ${ }^{47}$ There was no association with cardiovascular events based on a post hoc analysis of the Phase III SECURE trial. ${ }^{29,47}$ This was recently confirmed by a smaller study reporting that 24 out of 26 patients treated with isavuconazole developed a shortened QTc interval with a mean decrease of $36.5 \pm 38.8 \mathrm{~ms}(7.4 \% \pm 5.8 \%)$ compared to the pre-isavuconazole electrocardiogram. ${ }^{48}$

Thus, isavuconazole should be considered for use over the other azoles in patients on medications that have a known risk of QTc interval prolongation. Furthermore, isavuconazole could be an alternative in patients who develop azole-induced QTc interval prolongation. This approach has been reported in a recent case report of a 22-year-old woman with cystic fibrosis who developed voriconazole-associated QTc prolongation up to $613 \mathrm{~ms}$ in the absence of any 
electrolyte abnormality. Change from voriconazole to isavuconazole leads to a decrease in the QTc interval to $468 \mathrm{~ms}$ after 24 hours. ${ }^{74}$ However, patients with a known familial SQTS, which is a rare autosomal dominant inherited channelopathy, should not receive isavuconazole. In addition, isavuconazole can also be considered as an alternative antifungal treatment to other azole-induced adverse events including severe allergic reactions. ${ }^{75}$ Single case reports recommend a graded drug challenge with isavuconazole in case of previous azole-associated allergy. ${ }^{75,76}$

Based on a Phase I study, dose adjustment of isavuconazole is not required in patients with renal impairment or in patients who receive hemodialysis. ${ }^{77}$ Pharmacokinetic parameters were similar between healthy individuals and patients with mild, moderate, and severe renal impairment without a significant impact on renal function. In case of ESRD, isavuconazole can be administered before hemodialysis as isavuconazole is highly protein-bound ( $>98 \%$ to albumin) and unlikely to be cleared during hemodialysis. Conversely, hemodialysis cannot be used to clear isavuconazole in case of overdoses, whereas plasmapheresis may be a feasible option.

Current evidence suggests that for patients with IA or mucormycosis and mild to moderate hepatic impairment due to alcoholic cirrhosis or hepatitis $\mathrm{B} / \mathrm{C}$ virus infection, no dose adjustment of isavuconazole is needed..$^{78}$ Initially, dose adjustment of isavuconazole was recommended in patients with liver disease as data indicated that isavuconazole, which is primarily eliminated by hepatic metabolism, leads to a decreased systemic clearance after IV or oral administration and an increased half-life of isavuconazole in patients with mild and moderate liver impairment, compared to healthy individuals. ${ }^{79}$ However, based on a population pharmacokinetic model using data from this study, the authors revoked the recommendation later. ${ }^{78,79}$ Pharmacokinetic data in patients with severe hepatic impairment classified as Child-Pugh $\mathrm{C}$ are lacking. Dose adjustment is not necessary when changing from IV to oral formulations because oral isavuconazole is bioequivalent to the IV formulation. ${ }^{27}$

Based on EUCAST clinical breakpoints, isavuconazole is classified as susceptible in isolates with an MIC of $\leq 1 \mathrm{mg} / \mathrm{L} .{ }^{80}$ Recently published data indicate that highdose isavuconazole treatment (eg, $400 \mathrm{mg}$ once daily) might be an option in carefully selected patients infected with an A. fumigatus isolate with an isavuconazole MIC of $2 \mathrm{mg} / \mathrm{L} .{ }^{81}$ However, this study also showed that isavuconazole cannot be used for the treatment of patients with IA caused by an
A. fumigatus strain with TR34/L98H and TR46/Y121F/ T289A azole resistance mutations.

TDM may be considered in selected patients, especially when using high-dose isavuconazole treatment, but current clinical evidence does not recommend routine TDM for isavuconazole. ${ }^{82}$ Recently published data showed that the clinical dosing regimen achieved exposures adequate to treat IA in post hoc analyses of plasma isavuconazole concentrations of patients from the SECURE clinical trial. ${ }^{29,82}$

When compared to voriconazole, isavuconazole has been reported to be cost-effective for the first-line treatment of IA. ${ }^{83}$ Economic modeling assessed a cost saving of 7,418 US dollars per patient for isavuconazole compared to voriconazole. Astellas Pharma Global Development, Inc. funded this study. Evaluation was from a US hospital perspective using clinical data from the SECURE trial, including length of stay for the initial admission, incidence of readmission, clinical response, overall survival rates, and experience of adverse events.

\section{Conclusion and place in therapy}

Isavuconazole is a new second-generation triazole with a broad-spectrum antifungal activity and a favorable pharmacokinetic and safety profile, not requiring TDM. Compared to voriconazole and liposomal amphotericin B, it offers some advantages including good tolerability, a lower side effect profile, ${ }^{29}$ excellent bioavailability (switch from IV to oral treatment), ${ }^{27}$ and reduced drug-drug interactions. ${ }^{49,84,85}$ In the Phase III SECURE trial, isavuconazole demonstrated similar efficacy to voriconazole for the treatment of IA. ${ }^{29}$ Recent guidelines recommend isavuconazole as gold standard treatment (together with voriconazole) for IA in patients with hematological malignancies. ${ }^{30,31}$ In addition, isavuconazole has shown similar efficacy to liposomal amphotericin $\mathrm{B}$ for the treatment of infections from mucormycosis ${ }^{66}$ and is frequently used in real-life settings for the treatment of mucormycosis in patients who cannot tolerate liposomal amphotericin B (eg, reduced renal function). Updated guidelines on mucormycosis are needed to reflect the current evidence and give guidance on the use of isavuconazole for mucormycosis. Importantly, for empiric antifungal treatment, and treatment of suspected mold infections with unknown causative pathogen, isavuconazole and posaconazole may have favorable outcomes compared to voriconazole given their efficacy against mucorales.

Studies are needed to evaluate the role of isavuconazole for 1) anti-mold prophylaxis in high-risk patients; 2) treatment of IA in intensive care/solid-organ transplant/pulmonology 
settings, ie, in patients at risk but without an underlying hematological malignancy; 3) salvage treatment for IA and mucormycosis; and 4) treatment for other mold infections such as S. apiospermum.

\section{Author contributions}

JDJ, HJFS, JP, RK, DB, and MH performed the literature review and wrote the manuscript (each author was assigned one section). All the authors revised the manuscript critical for important intellectual content. All the authors approved the final version of the manuscript and agreed to be accountable for all aspects of the work in ensuring that questions related to the accuracy or integrity of any part of the work are appropriately investigated and resolved.

\section{Disclosure}

$\mathrm{MH}$ received research funding from Gilead and speakers honoraria from Gilead, Merck, and Basilea. HJFS received research funding from Gilead and speakers honoraria from Chiesi. JP is a consultant to Gilead. RK received research grants from Merck and served on the speakers' bureau of Pfizer, Gilead, Astellas, Basilea, and Merck. DB is a consultant to Basilea, Gilead Sciences, and Merck Sharp \& Dohme/ Merck; received research grants from Gilead Sciences and Pfizer; serves on the speakers' bureau of Astellas, Basilea, Gilead Sciences, Merck Sharp \& Dohme/Merck, Pfizer, and TEVA; and received travel grants from Astellas, Gilead Sciences, Merck Sharp \& Dohme/Merck, and Pfizer. The authors report no other conflicts of interest in this work.

\section{References}

1. Hoenigl M, Eigl S, Heldt S, Duettmann W, Thornton C, Prattes J. Clinical evaluation of the newly formatted lateral-flow device for invasive pulmonary aspergillosis. Mycoses. 2018;61(1):40-43.

2. Orasch T, Prattes J, Faserl K, et al. Bronchoalveolar lavage triacetylfusarinine $\mathrm{C}$ (TAFC) determination for diagnosis of invasive pulmonary aspergillosis in patients with hematological malignancies. J Infect. 2017; 75(4):370-373.

3. Eigl S, Prattes J, Lackner M, et al. Multicenter evaluation of a lateralflow device test for diagnosing invasive pulmonary aspergillosis in ICU patients. Crit Care. 2015;19:178.

4. Prattes J, Lackner M, Eigl S, et al. Diagnostic accuracy of the Aspergillusspecific bronchoalveolar lavage lateral-flow assay in haematological malignancy patients. Mycoses. 2015;58(8):461-469.

5. Hoenigl M, Prattes J, Spiess B, et al. Performance of galactomannan, beta-d-glucan, Aspergillus lateral-flow device, conventional culture, and PCR tests with bronchoalveolar lavage fluid for diagnosis of invasive pulmonary aspergillosis. J Clin Microbiol. 2014;52(6):2039-2045.

6. Eigl S, Hoenigl M, Spiess B, et al. Galactomannan testing and Aspergillus PCR in same-day bronchoalveolar lavage and blood samples for diagnosis of invasive aspergillosis. Med Mycol. 2017;55(5):528-534.

7. Heldt S, Eigl S, Prattes J, et al. Levels of IL-6, IL-8, IL-10 and IL-17A in serum and IL-8 in bronchoalveolar lavage fluid are elevated in haematological patients with invasive pulmonary aspergillosis. Mycoses. 2017; 60(12):818-825.
8. Prattes J, Flick H, Pruller F, et al. Novel tests for diagnosis of invasive aspergillosis in patients with underlying respiratory diseases. $\mathrm{Am} \mathrm{J}$ Respir Crit Care Med. 2014;190(8):922-929.

9. Eigl S, Prattes J, Reinwald M, et al. Influence of mould-active antifungal treatment on the performance of the Aspergillus-specific bronchoalveolar lavage fluid lateral-flow device test. Int $J$ Antimicrob Agents. 2015;46(4):401-405.

10. Hoenigl M, Prattes J, Neumeister P, Wolfler A, Krause R. Real-world challenges and unmet needs in the diagnosis and treatment of suspected invasive pulmonary aspergillosis in patients with haematological diseases: an illustrative case study. Mycoses. 2018;61(3):201-205.

11. Denning DW, Ribaud P, Milpied N, et al. Efficacy and safety of voriconazole in the treatment of acute invasive aspergillosis. Clin Infect Dis. 2002;34(5):563-571.

12. Cornely OA, Maertens J, Bresnik M, et al. Liposomal amphotericin B as initial therapy for invasive mold infection: a randomized trial comparing a high-loading dose regimen with standard dosing (AmBiLoad trial). Clin Infect Dis. 2007;44(10):1289-1297.

13. Herbrecht R, Denning DW, Patterson TF, et al. Voriconazole versus amphotericin B for primary therapy of invasive aspergillosis. $N$ Engl J Med. 2002;347(6):408-415.

14. Pagano L, Akova M, Dimopoulos G, Herbrecht R, Drgona L, Blijlevens N. Risk assessment and prognostic factors for mould-related diseases in immunocompromised patients. J Antimicrob Chemother. 2011; 66(Suppl 1):i5-i14.

15. Seyedmousavi S, Mouton JW, Verweij PE, Bruggemann RJ. Therapeutic drug monitoring of voriconazole and posaconazole for invasive aspergillosis. Expert Rev Anti Infect Ther. 2013;11(9):931-941.

16. Hoenigl M, Duettmann W, Raggam RB, et al. Potential factors for inadequate voriconazole plasma concentrations in intensive care unit patients and patients with hematological malignancies. Antimicrob Agents Chemother. 2013;57(7):3262-3267.

17. Prattes J, Duettmann W, Hoenigl M. Posaconazole plasma concentrations on days three to five predict steady-state levels. Antimicrob Agents Chemother. 2016;60(9):5595-5599.

18. Hoenigl M, Duettmann W, Raggam RB, et al. Impact of structured personal on-site patient education on low posaconazole plasma concentrations in patients with haematological malignancies. Int J Antimicrob Agents. 2014;44(2):140-144.

19. Vanstraelen K, Prattes J, Maertens J, et al. Posaconazole plasma exposure correlated to intestinal mucositis in allogeneic stem cell transplant patients. Eur J Clin Pharmacol. 2016;72(8):953-963.

20. Schmitt-Hoffmann A, Roos B, Heep M, et al. Single-ascending-dose pharmacokinetics and safety of the novel broad-spectrum antifungal triazole BAL4815 after intravenous infusions (50, 100, and 200 milligrams) and oral administrations (100, 200, and 400 milligrams) of its prodrug, BAL8557, in healthy volunteers. Antimicrob Agents Chemother. 2006;50(1):279-285.

21. Schmitt-Hoffmann A, Roos B, Maares J, et al. Multiple-dose pharmacokinetics and safety of the new antifungal triazole BAL4815 after intravenous infusion and oral administration of its prodrug, BAL8557, in healthy volunteers. Antimicrob Agents Chemother. 2006;50(1):286-293.

22. Donnelley MA, Zhu ES, Thompson GR 3rd. Isavuconazole in the treatment of invasive aspergillosis and mucormycosis infections. Infect Drug Resist. 2016;9:79-86.

23. Livermore J, Hope W. Evaluation of the pharmacokinetics and clinical utility of isavuconazole for treatment of invasive fungal infections. Expert Opin Drug Metab Toxicol. 2012;8(6):759-765.

24. Astellas Pharma US. Prescribing Information for Isavuconazonium Sulfate. Available from: https://www.astellas.us/docs/cresemba.pdf. Accessed April 6, 2018.

25. Schmitt-Hoffmann A, Roos B, Roerhle M. No Relevant Food Effect in Man on Isavuconazole Oral Pharmacokinetics Preliminary Data. Poster presented at: 48th Annual ICAAC/46th Annual IDSA; A-008; 2008. Available from: https://www.aspergillus.org.uk/content/no-relevantfood-effect-man-isavuconazole-oral-pharmacokinetics-preliminarydata. Accessed April 18, 2018. 
26. Kovanda LL, Marty FM, Maertens J, et al. Impact of mucositis on absorption and systemic drug exposure of isavuconazole. Antimicrob Agents Chemother. 2017;61(6):e101-e117.

27. Schmitt-Hoffmann A, Desai A, Kowalski D, Pearlman H, Yamazaki T, Townsend R. Isavuconazole absorption following oral administration in healthy subjects is comparable to intravenous dosing, and is not affected by food, or drugs that alter stomach $\mathrm{pH}$. Int J Clin Pharmacol Ther. 2016;54(8):572-580.

28. Kovanda LL, Desai AV, Lu Q, et al. Isavuconazole population pharmacokinetic analysis using nonparametric estimation in patients with invasive fungal disease (results from the VITAL study). Antimicrob Agents Chemother. 2016;60(8):4568-4576.

29. Maertens JA, Raad II, Marr KA, et al. Isavuconazole versus voriconazole for primary treatment of invasive mould disease caused by Aspergillus and other filamentous fungi (SECURE): a phase 3, randomisedcontrolled, non-inferiority trial. Lancet. 2016;387(10020):760-769.

30. Tissot F, Agrawal S, Pagano L, et al. ECIL-6 guidelines for the treatment of invasive candidiasis, aspergillosis and mucormycosis in leukemia and hematopoietic stem cell transplant patients. Haematologica. 2017;102(3):433-444.

31. Ullmann A, Aguado JM, Arikan S, et al. Executive summary of the 2017 ESCMID-ECMM guideline for the diagnosis and management of Aspergillus disease. Clin Microb Infect. 2018;24(1):e1-e38.

32. Hoenigl M, Raggam RB, Salzer HJ, et al. Posaconazole plasma concentrations and invasive mould infections in patients with haematological malignancies. Int J Antimicrob Agents. 2012;39(6):510-513.

33. Schmitt-Hoffman A, Richter WF. Isavuconazole Is Widely Distributed in Rat Tissue. ECCMID, Poster P863; 2012.

34. Everson N, Smith J, Garner D. Successful Treatment of Contaminated Epidural Steroid Associated Fungal Meningitis with Isavuconazole. ECCMID, P0231; 2015.

35. Yamazaki T, Inagaki Y, Fujii T, et al. In vitro activity of isavuconazole against 140 reference fungal strains and 165 clinically isolated yeasts from Japan. Int J Antimicrob Agents. 2010;36(4):324-331.

36. Pfaller MA, Rhomberg PR, Messer SA, Jones RN, Castanheira M. Isavuconazole, micafungin, and 8 comparator antifungal agents' susceptibility profiles for common and uncommon opportunistic fungi collected in 2013: temporal analysis of antifungal drug resistance using CLSI species-specific clinical breakpoints and proposed epidemiological cutoff values. Diagn Microbiol Infect Dis. 2015;82(4):303-313.

37. Seifert H, Aurbach U, Stefanik D, Cornely O. In vitro activities of isavuconazole and other antifungal agents against $C$ andida bloodstream isolates. Antimicrob Agents Chemother. 2007;51(5):1818-1821.

38. Pfaller MA, Messer SA, Rhomberg PR, Jones RN, Castanheira M In vitro activities of isavuconazole and comparator antifungal agents tested against a global collection of opportunistic yeasts and molds. J Clin Microbiol. 2013;51(8):2608-2616.

39. Thompson GR 3rd, Wiederhold NP, Fothergill AW, Vallor AC, Wickes BL, Patterson TF. Antifungal susceptibilities among different serotypes of Cryptococcus gattii and Cryptococcus neoformans. Antimicrob Agents Chemother. 2009;53(1):309-311.

40. Guinea J, Recio S, Escribano P, Pelaez T, Gama B, Bouza E. In vitro antifungal activities of isavuconazole and comparators against rare yeast pathogens. Antimicrob Agents Chemother. 2010;54(9):4012-4015.

41. Natesan SK, Chandrasekar PH. Isavuconazole for the treatment of invasive aspergillosis and mucormycosis: current evidence, safety, efficacy, and clinical recommendations. Infect Drug Resist. 2016;9:291-300.

42. Warn PA, Sharp A, Mosquera J, et al. Comparative in vivo activity of BAL4815, the active component of the prodrug BAL8557, in a neutropenic murine model of disseminated Aspergillus flavus. J Antimicrob Chemother. 2006;58(6):1198-1207.

43. Datta K, Rhee P, Byrnes E 3rd, et al. Isavuconazole activity against Aspergillus lentulus, Neosartorya udagawae, and Cryptococcus gattii, emerging fungal pathogens with reduced azole susceptibility. J Clin Microbiol. 2013;51(9):3090-3093.

44. Gonzalez GM. In vitro activities of isavuconazole against opportunistic filamentous and dimorphic fungi. Med Mycol. 2009;47(1):71-76.
45. Guinea J, Pelaez T, Recio S, Torres-Narbona M, Bouza E. In vitro antifungal activities of isavuconazole (BAL4815), voriconazole, and fluconazole against 1,007 isolates of zygomycete, Candida, Aspergillus, Fusarium, and Scedosporium species. Antimicrob Agents Chemother. 2008;52(4):1396-1400.

46. Viljoen J, Azie N, Schmitt-Hoffmann AH, Ghannoum M. A phase 2, randomized, double-blind, multicenter trial to evaluate the safety and efficacy of three dosing regimens of isavuconazole compared with fluconazole in patients with uncomplicated esophageal candidiasis. Antimicrob Agents Chemother. 2015;59(3):1671-1679.

47. Keirns J, Desai A, Kowalski D, et al. QT interval shortening with isavuconazole: in vitro and in vivo effects on cardiac repolarization. Clin Pharmacol Ther. 2017;101(6):782-790.

48. Mellinghoff SC, Bassetti M, Dorfel D, et al. Isavuconazole shortens the QTc interval. Mycoses. 2017;61(4):256-260.

49. Groll AH, Desai A, Han D, et al. Pharmacokinetic assessment of drug-drug interactions of isavuconazole with the immunosuppressants cyclosporine, mycophenolic acid, prednisolone, sirolimus, and tacrolimus in healthy adults. Clin Pharmacol Drug Dev. 2017;6(1):76-85.

50. Cornely OA, Lass-Florl C, Lagrou K, Arsic-Arsenijevic V, Hoenigl M. Improving outcome of fungal diseases - guiding experts and patients towards excellence. Mycoses. 2017;60(7):420-425.

51. Petraitis V, Petraitiene R, Moradi PW, et al. Pharmacokinetics and concentration-dependent efficacy of isavuconazole for treatment of experimental invasive pulmonary aspergillosis. Antimicrob Agents Chemother. 2016;60(5):2718-2726.

52. U.S. Food and Drug Administration. Drug Approval Package: CRESEMBA (Isavuconazonium Sulfate; BAL8557). FDA Application NDA 207500 and 207501. 2014.

53. Lepak AJ, Marchillo K, Vanhecker J, Andes DR. Isavuconazole (BAL4815) pharmacodynamic target determination in an in vivo murine model of invasive pulmonary aspergillosis against wild-type and cyp51 mutant isolates of Aspergillus fumigatus. Antimicrob Agents Chemother. 2013;57(12):6284-6289.

54. Majithiya JB, Sharp A, Parmar A, Denning DW, Warn PA. Correlation of In Vitro MIC and MFC against Isavuconazole, Voriconazole and Amphotericin B of Aspergillus with In Vivo Outcome in Mice with Disseminated Aspergillosis. Barcelona, Spain: 2008.

55. Cornely OA, Bohme A, Schmitt-Hoffmann A, Ullmann AJ. Safety and pharmacokinetics of isavuconazole as antifungal prophylaxis in acute myeloid leukemia patients with neutropenia: results of a phase 2 , dose escalation study. Antimicrob Agents Chemother. 2015;59(4): 2078-2085.

56. Patterson TF, Thompson GR 3rd, Denning DW, et al. Executive summary: practice guidelines for the diagnosis and management of aspergillosis: 2016 update by the infectious diseases society of America. Clin Infect Dis. 2016;63(4):433-442.

57. Petrikkos G, Skiada A, Lortholary O, Roilides E, Walsh TJ, Kontoyiannis DP. Epidemiology and clinical manifestations of mucormycosis. Clin Infect Dis. 2012;54(Suppl 1):S23-S34.

58. Cornely OA, Arikan-Akdagli S, Dannaoui E, et al. ESCMID and ECMM joint clinical guidelines for the diagnosis and management of mucormycosis 2013. Clin Microbiol Infect. 2014;20(Suppl 3):5-26.

59. Skiada A, Lanternier F, Groll AH, et al. Diagnosis and treatment of mucormycosis in patients with hematological malignancies: guidelines from the 3rd European Conference on Infections in Leukemia (ECIL 3). Haematologica. 2013;98(4):492-504.

60. Spellberg B, Ibrahim AS, Chin-Hong PV, et al. The deferasiroxAmBisome therapy for mucormycosis (DEFEAT Mucor) study: a randomized, double-blinded, placebo-controlled trial. J Antimicrob Chemother. 2012;67(3):715-722.

61. Lanternier F, Lortholary O. AMBIZYGO: phase II study of high dose liposomal amphotericin B (AmBisome) $[10 \mathrm{mg} / \mathrm{kg} / \mathrm{j}]$ efficacy against zygomycosis. Med Mal Infect. 2008;38(Suppl 2):S90-S91.

62. Arendrup MC, Jensen RH, Meletiadis J. In vitro activity of isavuconazole and comparators against clinical isolates of the mucorales order. Antimicrob Agents Chemother. 2015;59(12):7735-7742. 
63. Luo G, Gebremariam T, Lee H, Edwards JE Jr, Kovanda L, Ibrahim AS. Isavuconazole therapy protects immunosuppressed mice from mucormycosis. Antimicrob Agents Chemother. 2014;58(4):2450-2453.

64. Thompson GR 3rd, Wiederhold NP. Isavuconazole: a comprehensive review of spectrum of activity of a new triazole. Mycopathologia. 2010;170(5):291-313.

65. Nucci M, Marr KA, Vehreschild MJ, et al. Improvement in the outcome of invasive fusariosis in the last decade. Clin Microbiol Infect. 2013;20(6):580-585.

66. Marty FM, Ostrosky-Zeichner L, Cornely OA, et al. Isavuconazole treatment for mucormycosis: a single-arm open-label trial and casecontrol analysis. Lancet Infect Dis. 2016;16(7):828-837.

67. Kara IO, Tasova Y, Uguz A, Sahin B. Mucormycosis-associated fungal infections in patients with haematologic malignancies. Int J Clin Pract. 2009;63(1):134-139.

68. Peixoto D, Gagne LS, Hammond SP, et al. Isavuconazole treatment of a patient with disseminated mucormycosis. J Clin Microbiol. 2014; 52(3):1016-1019.

69. Ervens J, Ghannoum M, Graf B, Schwartz S. Successful isavuconazole salvage therapy in a patient with invasive mucormycosis. Infection. 2014;42(2):429-432.

70. Graves B, Morrissey CO, Wei A, et al. Isavuconazole as salvage therapy for mucormycosis. Med Mycol Case Rep. 2016;11:36-39.

71. Hoenigl M, Krause R. Antifungal therapy of aspergillosis of the central nervous system and Aspergillus endophthalmitis. Curr Pharm Des. 2013;19(20):3648-3668.

72. Reischies F, Hoenigl M. The role of surgical debridement in different clinical manifestations of invasive aspergillosis. Mycoses. 2014; 57(Suppl 2):1-14

73. Mellinghoff SC, Panse J, Alakel N, et al. Primary prophylaxis of invasive fungal infections in patients with haematological malignancies: 2017 update of the recommendations of the Infectious Diseases Working Party (AGIHO) of the German Society for Haematology and Medical Oncology (DGHO). Ann Hematol. 2018;97(2): 197-207.

74. Trang TP, Hanretty AM, Langelier C, Yang K. Use of isavuconazole in a patient with voriconazole-induced QTc prolongation. Transpl Infect Dis. Epub 2017 Jun 20;19(4):doi:10.1111/tid.12712.

75. Morales MK, Harris C, Shoham S. Graded isavuconazole introduction in a patient with voriconazole allergy. Transpl Infect Dis. Epub 2017 Nov 3;19(6):doi:10.1111/tid.12772.

76. Benjamin Lash D, Jolliff J, Munoz A, Heidari A. Cross-reactivity between voriconazole, fluconazole and itraconazole. J Clin Pharm Ther. 2016;41(5):566-567.
77. Townsend RW, Akhtar S, Alcorn H, et al. Phase I trial to investigate the effect of renal impairment on isavuconazole pharmacokinetics. Eur J Clin Pharmacol. 2017;73(6):669-678.

78. Desai A, Schmitt-Hoffmann AH, Mujais S, Townsend R. Population pharmacokinetics of isavuconazole in subjects with mild or moderate hepatic impairment. Antimicrob Agents Chemother. 2016;60(5): 3025-3031.

79. Schmitt-Hoffmann A, Roos B, Spickermann J, et al. Effect of mild and moderate liver disease on the pharmacokinetics of isavuconazole after intravenous and oral administration of a single dose of the prodrug BAL8557. Antimicrob Agents Chemother. 2009;53(11):4885-4890.

80. Arendrup MC, Meletiadis J, Mouton JW, et al. EUCAST technical note on isavuconazole breakpoints for Aspergillus, itraconazole breakpoints for Candida and updates for the antifungal susceptibility testing method documents. Clin Microbiol Infect. 2016;22(6):571.e1-571.e4.

81. Buil JB, Bruggemann RJM, Wasmann RE, et al. Isavuconazole susceptibility of clinical Aspergillus fumigatus isolates and feasibility of isavuconazole dose escalation to treat isolates with elevated MICs. J Antimicrob Chemother. 2018;73(1):134-142.

82. Desai AV, Kovanda LL, Hope WW, et al. Exposure-response relationships for isavuconazole in patients with invasive aspergillosis and other filamentous fungi. Antimicrob Agents Chemother. 2017;61(12): e01034-e01117. Available from: http://aac.asm.org/content/61/12/ e01034-17.full. Accessed April 18, 2018.

83. Harrington R, Lee E, Yang H, et al. Cost-effectiveness analysis of isavuconazole vs. voriconazole as first-line treatment for invasive aspergillosis. Adv Ther. 2017;34(1):207-220.

84. Townsend R, Dietz A, Hale C, et al. Pharmacokinetic evaluation of CYP3A4-mediated drug-drug interactions of isavuconazole with rifampin, ketoconazole, midazolam, and ethinyl estradiol/norethindrone in healthy adults. Clin Pharmacol Drug Dev. 2017;6(1):44-53.

85. Yamazaki T, Desai A, Goldwater R, et al. Pharmacokinetic effects of isavuconazole coadministration with the cytochrome P450 enzyme substrates bupropion, repaglinide, caffeine, dextromethorphan, and methadone in healthy subjects. Clin Pharmacol Drug Dev. 2017;6(1): 54-65.

86. Castanheira M, Messer SA, Rhomberg PR, Dietrich RR, Jones RN, Pfaller MA. Isavuconazole and nine comparator antifungal susceptibility profiles for common and uncommon Candida species collected in 2012: application of new CLSI clinical breakpoints and epidemiological cutoff values. Mycopathologia. 2014;178(1-2):1-9.

87. Thompson GR 3rd, Rendon A, Ribeiro dos Santos R, et al. Isavuconazole treatment of cryptococcosis and dimorphic mycoses. Clin Infect Dis. 2016;63(3):356-362.
Drug Design, Development and Therapy

\section{Publish your work in this journal}

Drug Design, Development and Therapy is an international, peerreviewed open-access journal that spans the spectrum of drug design and development through to clinical applications. Clinical outcomes, patient safety, and programs for the development and effective, safe, and sustained use of medicines are the features of the journal, which

\section{Dovepress}

has also been accepted for indexing on PubMed Central. The manuscript management system is completely online and includes a very quick and fair peer-review system, which is all easy to use. Visit http://www.dovepress.com/testimonials.php to read real quotes from published authors. 